\title{
A New Approach to the Analysis of Vessel Residence Time Distribution Curves
}

\author{
SERGIO P. FERRO, R. JAVIER PRINCIPE, and MARCELA B. GOLDSCHMIT \\ Mathematical models for the evaluation of residence time distribution (RTD) curves on a large variety \\ of vessels are presented. These models have been constructed by combination of different tanks or \\ volumes. In order to obtain a good representation of RTD curves, a new volume (called convection \\ diffusion volume) is introduced. The convection-diffusion volume allows the approximation of different \\ experimental or numerical RTD curves with very simple models. An algorithm has been developed \\ to calculate the parameters of the models for any given set of RTD curve experimental points. \\ Validation of the models is carried out by comparison with experimental RTD curves taken from the \\ literature and with a numerical RTD curve obtained by three-dimensional simulation of the flow \\ inside a tundish.
}

\section{INTRODUCTION}

THE residence time of an element of fluid is the time it spends inside the vessel. Since different elements of fluid spend different times inside the vessel, there is a distribution of residence times for each vessel. The residence time distribution (RTD) gives important information about the behavior of the flow inside the different vessels of the continuous caster. Both experimental and numerical techniques can be employed to obtain the RTD. ${ }^{[1]}$ In both cases, the basic idea is to inject a pulse of a tracer at the entrance of the vessel and to analyze the concentration of tracer at the exit as a function of time. This function is known as the RTD curve. The RTD curves are generally represented in terms of dimensionless variables (to be defined in the following section) and typically take the shape of the curve depicted in Figure 1.

Experimental RTD curves are obtained in water models by the injection of a salt solution or a dye as a tracer. In the former case, the concentration at the exit of the vessel is obtained by measuring the conductivity of the fluid. In the latter, colorimetry or spectrophotometry techniques are used to obtain the concentration.

The RTD curves can also be obtained by numerical methods. In this case, the first step is to calculate the turbulent flow inside the vessel. Different techniques could be applied to solve the steady-state turbulent Navier-Stokes equations. The $k-\varepsilon$ method, where $k$ is the turbulent kinetic energy and $\varepsilon$ is the turbulent kinetic energy dissipation rate, is the most popular. ${ }^{[2]}$ Once the velocity distribution inside the vessel is obtained, the addition of tracer needs to be numerically simulated. A turbulent convection diffusion equation for the concentration of tracer must be solved. A narrow step function has to be imposed at the entrance as a boundary condition to simulate the tracer pulse injection. The RTD curve is the concentration of tracer at the exit of the vessel as a function of time.

A different approach to the analysis of residence times

SERGIO P. FERRO and R. JAVIER PRINCIPE, Researchers, and MARCELA B. GOLDSCHMIT, Head of Computational Mechanics Department, are with the Center for Industrial Research (CINI), FUDETEC, 1054 Buenos Aires, Argentina.

Manuscript submitted May 14, 2001. inside vessels is the numerical modeling by tanks or volumes. ${ }^{[3]}$ In these models, the vessel is divided into different regions (the tanks or volumes) where the flow is supposed to behave in a very simple way. For each volume, the concentration evolves according to a specific differential equation. The models presented in this article are based on this method.

The numerical modeling of the flow inside vessels by tanks or volumes is widely used in the literature, as described in an extensive review published by Mazumdar and Guthrie. ${ }^{[1]}$ Many of these works present models obtained by combinations of mixing volumes, plug flow volumes, and dead volumes. ${ }^{[3]}$ However, a good description of RTD curves in terms of these models (called mixed flow models) is not always possible. To improve the accuracy of the models, different modifications were introduced. Martin, ${ }^{[4]}$ for instance, proposed a modification of the tank in series model (where the system is divided into several identical mixing volumes) by considering a noninteger number of tanks. Sahai and Ahuja, ${ }^{[5]}$ on the other hand, introduced the use of dispersed plug flow volumes instead of the standard plug flow volumes in their study of tundish RTD curves.

The dispersed plug flow volume is based on the dispersion model introduced by Levenspiel and Smith ${ }^{[6]}$ in the chemical reactor analysis and represents a deviation from the ideal plug flow caused by longitudinal mixing. In the dispersion model, the evolution of tracer concentration in any vessel is modeled by the one-dimensional transient convectiondiffusion equation. Dispersion models describe accurately the mixing process if the diffusivity is small, but are deficient when diffusivity is large. ${ }^{[3,7]}$

In Section II, we introduce a new kind of volume named convection-diffusion volume. This volume is also based on dispersion models and is able to describe RTD curves in vessels with any amount of diffusion. We comment on the physical interpretation of this new convection-diffusion volume and explain its difference with the dispersed plug flow volume. In Section III, we propose a simple model consisting of a single convection-diffusion volume and a dead volume: experimental RTD curves found in the literature are analyzed in terms of this model. In order to represent two peaked RTD curves, another model is proposed in Section IV, which consists of two convection diffusion volumes and a dead volume. This model is also used to analyze experimental 


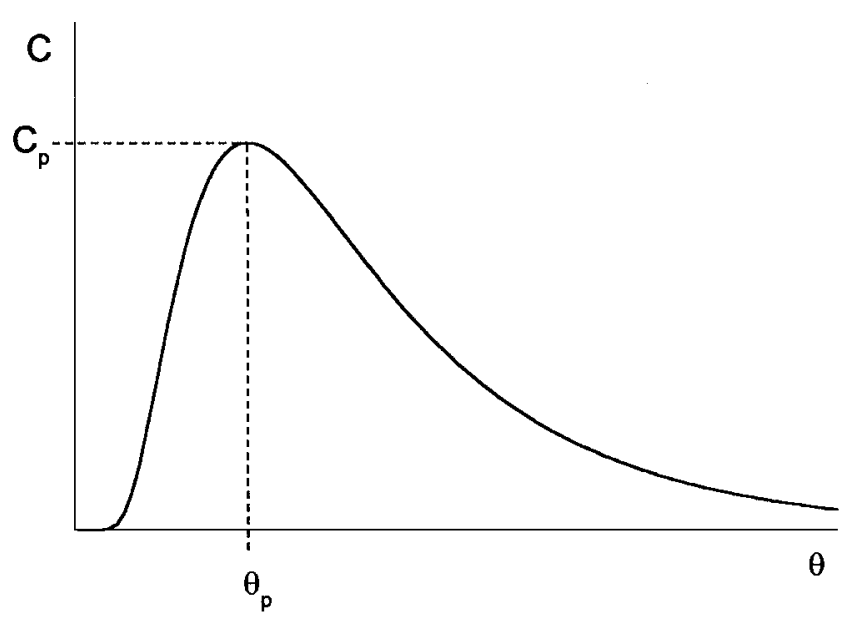

Fig. 1-Typical RTD curve.

data, especially two peaked RTD curves. In Section V, we present a three-dimensional numerical analysis of a four line tundish and the interpretation of its RTD curves in terms of the model deseribed in Section IV. The last section is devoted to conclusions.

II. CONVECTION-DIFFUSION VOLUMES

A. Basic Equations

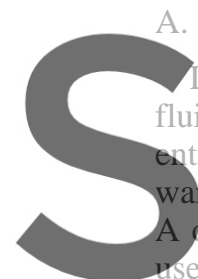

Let us consider a system w fluid enters and exits the system entrance of this system, a trader want to describe the concent ed to represent the evolution of concentration of tracer inside the volume,

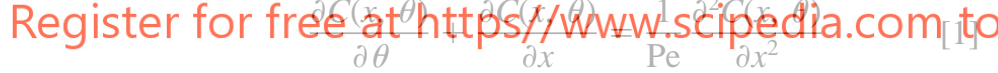

where $C(x, \theta)$ is the dimensionless tracer concentration and $x$ and $\theta$ are the dimensionless time and position expressed in terms of the length of the domain, $L$, and the theoretical residence time $\tau=\frac{V}{Q}=\frac{L}{v}$ ( $v$ is the velocity inside the domain, assumed constant). The term Pe is the turbulent Péclet number, $\mathrm{Pe}=\frac{v L}{D}$, where $D$ is the turbulent diffusivity.

We want to solve this equation for all $0<x \leq 1$ and $\theta>$ 0 according to the following initial and boundary conditions:

$$
\begin{aligned}
& C(x, 0)=0 \\
& C(0, \theta)=C_{0}(\theta)
\end{aligned}
$$

where $C_{0}(\theta)$ is any given function of time.

The solution of Eq. [1], which satisfies condition [2] and does not diverge for large values of $x$, is

$$
C(x, \theta)=\int_{0}^{\theta} K_{\mathrm{Pe}}(x, \theta-\tau) C_{0}(\tau) d \tau
$$

where the kernel $K_{\mathrm{Pe}}(x, \theta)$ is defined by

$$
K_{\mathrm{Pe}}(x, \theta)=x \sqrt{\frac{\mathrm{Pe}}{4 \pi \theta^{3}}} \exp \left(-\frac{\mathrm{Pe}(x-\theta)^{2}}{4 \theta}\right)
$$

The kernel satisfies the differential equation, the initial condition, and the integral property $\int_{0}^{\infty} K_{\mathrm{Pe}}(x, \theta) d \theta=1$ for all $x>0$. Since the kernel vanishes at $x=0$ for all $\theta>0$, the boundary condition for the kernel may be written as $K_{\mathrm{Pe}}(0$, $\theta)=\delta(\theta)$, where $\delta(\theta)$ is Dirac's delta function. From these properties of the kernel, it is possible to show that $C(x, \theta)$ satisfies Eq. [1] with condition [2].

We are interested in the concentration of tracer at the outlet of volume $x=1$.

$$
C(1, \theta)=\int_{0}^{\theta} K_{\mathrm{Pe}}(1, \theta-\tau) C_{0}(\tau) d \tau
$$

with $K_{\mathrm{Pe}}(1, \theta)$ given by

$$
K_{\mathrm{Pe}}(1, \theta)=\sqrt{\frac{\mathrm{Pe}}{4 \pi \theta^{3}}} \exp \left(-\frac{\operatorname{Pe}(1-\theta)^{2}}{4 \theta}\right)
$$

Now, we introduce the convection-diffusion volume defined by its volume $V$ and its Péclet number Pe. In this volume, if the concentration at the entrance $C_{0}(\theta)$ and the flow rate $Q$ are given, the concentration at the exit is calculated from Eq. [3].

\section{B. Physical Interpretation}

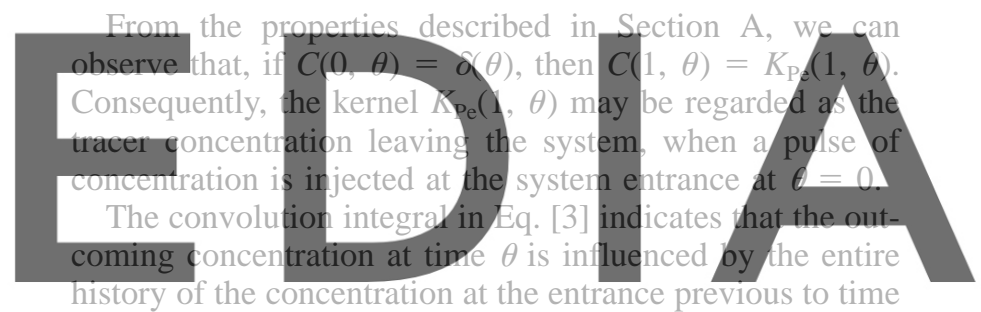
$\theta$. Also, since the kernel at time $\theta$ is the response of the

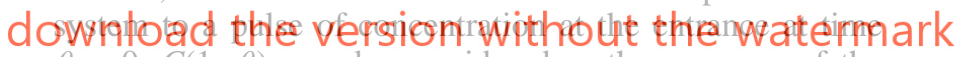
$\theta=0, C(1, \theta)$ may be considered as the response of the system to a series of pulses of amplitude $C(0, \tau) d \tau$ injected at the entrance at time $\tau$.

The set of equations described in Section A were deduced under the assumption of an open system. That is, the diffusivity is considered continuous across the input-output boundaries of the system. ${ }^{[7]}$

The Péclet number indicates how diffusive the flow in the system is.

(1) The limit $\mathrm{Pe} \rightarrow \infty$ corresponds to purely convective flow with no diffusion. In this limit, $K_{\mathrm{Pe}}(1, \theta) \rightarrow \delta(1-\theta)$ and $C(1, \theta) \rightarrow C(0, \theta-1)$. This is the expected solution for the concentration of tracer in a system with no diffusion, that is, a plug flow system.

(2) On the other hand, when $\mathrm{Pe} \rightarrow 0$, diffusion is very large and the concentration at the entrance propagates instantaneously along the volume. The function $K_{\mathrm{Pe}}(1, \theta)$ presents a sharp peak near $\theta=0$ vanishing anywhere else and $C(1, \theta) \rightarrow C(0, \theta)$. This result contrasts with the large diffusivity limit for closed systems

$$
C(1, \theta) \rightarrow \int_{0}^{\theta} e-(\theta-\tau) C_{0}(\tau) d \tau^{[7]}
$$

Let's analyze the behavior of $C(1, \theta)$ in a convectiondiffusion volume when a pulse is injected at the entrance. In this case, $C(1, \theta)=K_{\mathrm{Pe}}(1, \theta)$ is the RTD curve of a 


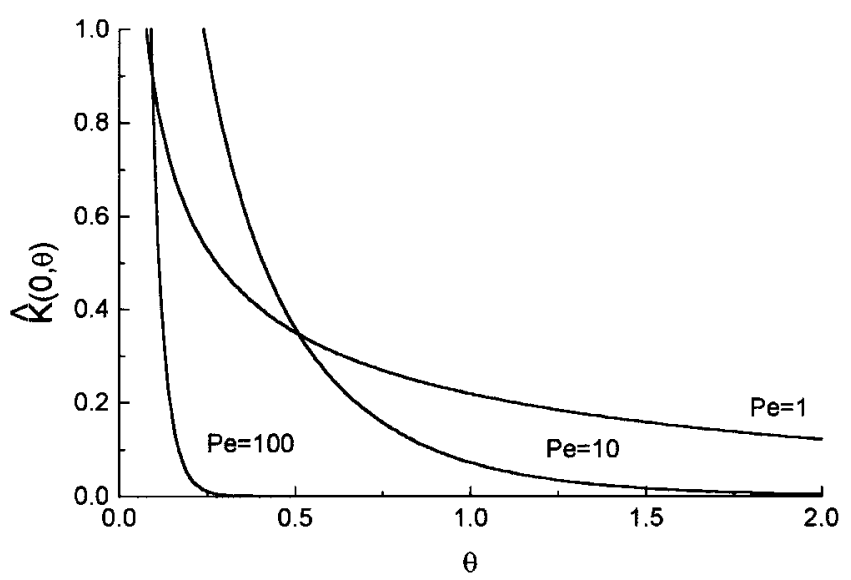

Fig. 2-Concentration at the origin as a function of time, for the solution of Eq. [1] described in Ref. 6.

system represented by a single convection-diffusion volume. Since $C(1,0)=0$ (initial condition, Eq. [2]) and $C(1, \theta)$ $\rightarrow 0$ as $\theta \rightarrow \infty$ (from Eq. [4]), the curve must reach a maximum value $C_{p}$ at a certain time $\theta_{p}$. The general appearance of such a curve has already been shown in Figure 1. The values of $\theta_{p}$ and $C_{p}$ are given by

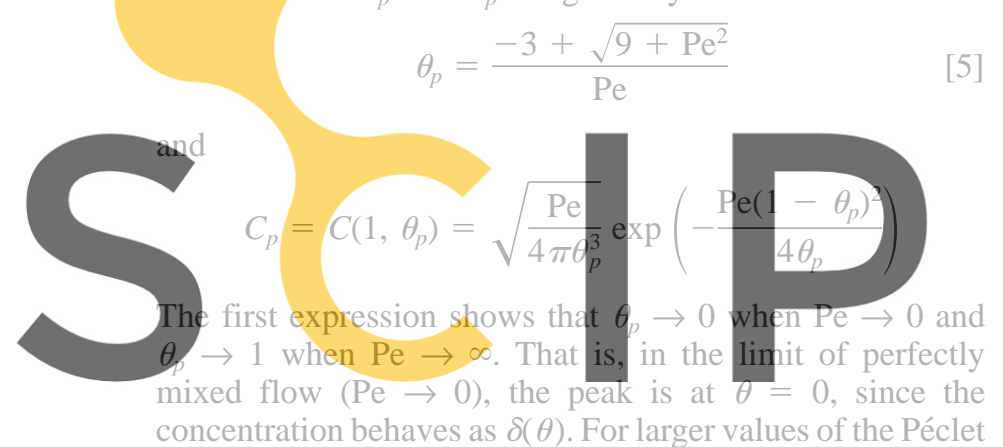

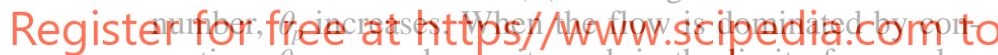
vection, $\theta_{p}$ approaches unity and, in the limit of pure plug flow, $\theta_{p}=1$, in agreement with the fact that, in this limit, the concentration tends toward $\delta(1-\theta)$. In consequence, the Péclet number can be estimated from the position of the RTD curve peak,

$$
\mathrm{Pe}=\frac{6 \theta_{p}}{1-\theta_{p}^{2}}
$$

\section{Comments on Boundary Condition}

It is important to note that the dispersion model of Levenspiel and Smith makes use of a rather different function to describe the response of the system to a pulse injected at the entrance, ${ }^{[6]}$

$$
\hat{K}_{\mathrm{Pe}}(x, \theta)=\sqrt{\frac{\mathrm{Pe}}{4 \pi \theta}} \exp \left(-\frac{\operatorname{Pe}(x-\theta)^{2}}{4 \theta}\right)=\frac{\theta}{x} K_{\mathrm{Pe}}(x, \theta)
$$

The kernel $\hat{K}_{\mathrm{Pe}}(x, \theta)$ was obtained from a different solution of Eq. [1]. ${ }^{[6]}$ At the entrance, this kernel does not represent a pulse, since it does not vanish for $\theta>0$,

$$
\hat{K}_{\mathrm{Pe}}(0, \theta)=\sqrt{\frac{\mathrm{Pe}}{4 \pi \theta}} \exp \left(-\frac{\mathrm{Pe} \theta}{4}\right)
$$

In Figure 2, the function $\hat{K}_{\mathrm{Pe}}(0, \theta)$ is plotted as function of $\theta$ for different values of the parameter Pe. For large values of the Péclet number (i.e., if diffusion is low), the concentration decreases rapidly away from $\theta=0$, and in the limit $\mathrm{Pe} \rightarrow \infty$, $\hat{K}_{\mathrm{Pe}}(0, \theta)$ tends to form of a pulse. However, for general values of $\mathrm{Pe}, \hat{K}(0, \theta)$ presents long tails. This means that the tracer is injected at the entrance of the system during a certain period of time instead of being injected instantaneously at $\theta=0$. The function $\hat{K}_{\mathrm{Pe}}(x, \theta)$ can be regarded as the response of the system to a pulse at the entrance, but only in the limit of plug flow. It is easy to see that in this limit,

$$
K_{\mathrm{Pe}}(1, \theta) \approx \hat{K}_{\mathrm{Pe}}(1, \theta) \approx \sqrt{\frac{\mathrm{Pe}}{4 \pi}} \exp \left(-\frac{\mathrm{Pe}(1-\theta)^{2}}{4}\right)
$$

It is also interesting to compare the average residence times, $\theta_{a v}=\int_{0}^{\infty} \theta K_{\mathrm{Pe}}(1, \theta) d \theta$ and $\hat{\theta}_{a v}=\int_{0}^{\infty} \theta \hat{K}_{\mathrm{Pe}}(1, \theta) d \theta$ It can be seen that $\hat{\theta}_{a v}=1+\frac{2}{\mathrm{Pe}}^{[6]}$ only remains close to the unity in the limit of plug flow and diverges on the limit of high diffusion. On the other hand, $\theta_{a v}=1$ for any value of Pe.

As pointed out in References 3 and 7, the dispersion model can be used only for systems with a relatively small degree of mixing. It is our belief that the use of the kernel $K_{\mathrm{Pe}}(x, \theta)$ instead of $\hat{K}_{\mathrm{Pe}}(x, \theta)$ allows the application of the dispersion model to more general situations.

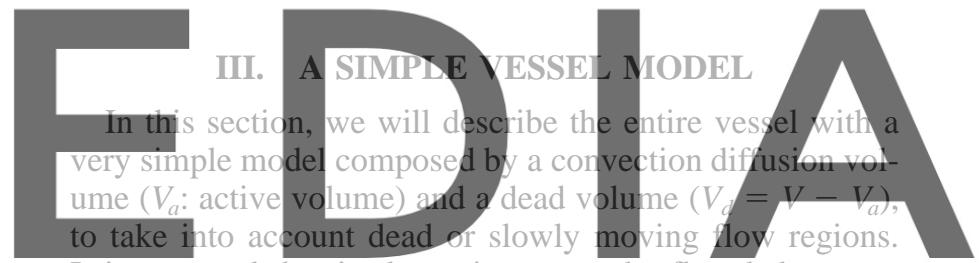

It is assumed that in the active zone, the flow behaves as one dimensional with a characteristic time $V_{a} / Q$. When the

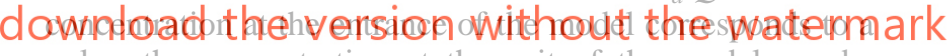
pulse, the concentration at the exit of the model can be obtained from Eq. [3].

$$
C(1, \theta)=\frac{1}{v_{a}} K_{\mathrm{Pe}}\left(1, \frac{\theta}{v_{a}}\right)=\sqrt{\frac{v_{a} \mathrm{Pe}}{4 \pi \theta^{3}}} \exp \left(-\frac{\operatorname{Pe}\left(v_{a}-\theta\right)^{2}}{4 v_{a} \theta}\right)
$$

which is the mathematical expression for the RTD curve. This curve reaches its maximum when

$$
\theta_{p}=v_{a} \frac{-3+\sqrt{9+\mathrm{Pe}^{2}}}{\mathrm{Pe}}
$$

The model has two dimensionless parameters, the Péclet number Pe and the fraction of the active volume $v_{a}=V_{a} / V$. It is useful to relate these parameters to the values of $\theta_{p}$, $C_{p}$ and $\theta_{a v}$ (where $\theta_{a v}=\int_{0}^{\infty} \theta C(1, \theta) d \theta$ is the average residence time). The following expressions were obtained:

$$
\begin{aligned}
v_{a} & =\theta_{a v} \\
v_{d} & =1-\theta_{a v} \\
\mathrm{Pe} & =\frac{6 \theta_{p} \theta_{a v}}{\theta_{a v}^{2}-\theta_{p}^{2}}
\end{aligned}
$$

Then, the parameters of the simple vessel model $v_{a}$ and 


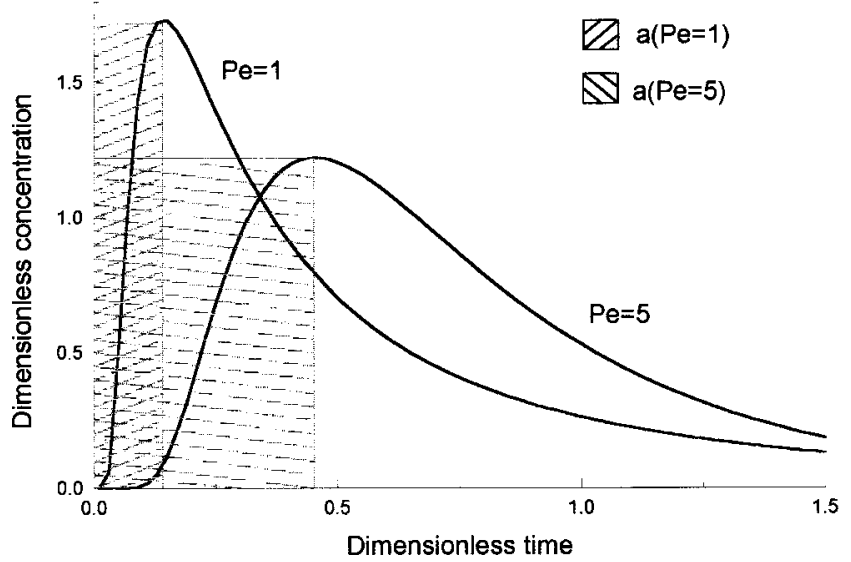

Fig. 3-Relationship between the location of the peak of the RTD curve and the function $a(\mathrm{Pe})$.

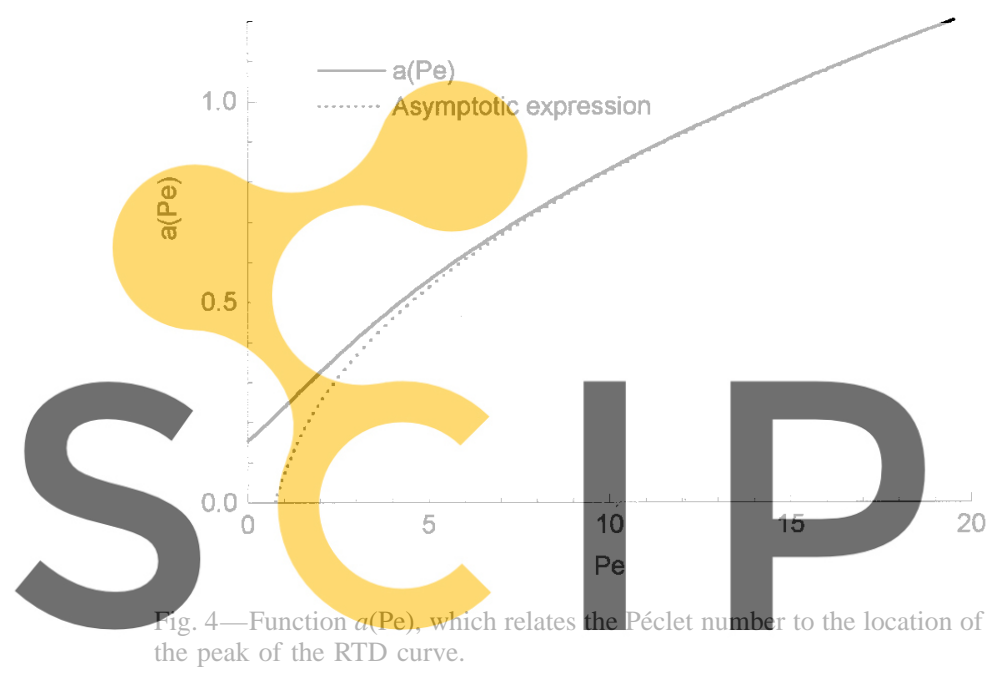

Register for free at https//www.scipedia.com to experimental RTD curve.

However, the numerical calculation of $\theta_{a v}$ from experimental curves can be difficult, especially for RTD curves with very long tails. In order to find another way to estimate the parameters of the model, the product $C_{p} \theta_{p}$ is going to be considered. This product does not depend on $v_{a}$ and is only a function of Pe:

$$
\begin{aligned}
C_{p} \theta_{p}=a(\mathrm{Pe}) & =\frac{\mathrm{Pe}}{\sqrt{4 \pi\left(-3+\sqrt{9+\mathrm{Pe}^{2}}\right)}} \\
& \exp \left[-\frac{\left(\mathrm{Pe}+3-\sqrt{9+\mathrm{Pe}^{2}}\right)^{2}}{4\left(-3+\sqrt{9+\mathrm{Pe}^{2}}\right)}\right]
\end{aligned}
$$

In an RTD diagram, $a(\mathrm{Pe})$ represents the rectangular area subtended by the origin and the peak of the curve (Figure 3) and is a monotonous increasing function of the Pe (Figure 4). We found that $a(\mathrm{Pe}) \rightarrow a_{0} \simeq 0.154$ when $\mathrm{Pe} \rightarrow 0$ and that $a(\mathrm{Pe}) \rightarrow \sqrt{\frac{\mathrm{Pe}}{4 \pi}}\left(1-\frac{3}{4 \mathrm{Pe}}\right)$ when $\mathrm{Pe} \rightarrow \infty$. This asymptotic expression can be inverted to obtain a good estimation of Pe when the flow has a small degree of diffusion:

$$
\mathrm{Pe} \approx \frac{3}{4}+2 \pi\left(C_{p} \theta_{p}\right)^{2}\left(1-\sqrt{1+\frac{3}{4 \pi\left(C_{p} \theta_{p}\right)^{2}}}\right)
$$

Table I. Cases Considered for the Validation of the Simple Vessel Model

\begin{tabular}{lcc}
\hline \multicolumn{1}{c}{ Case } & 1 & 2 \\
\hline Authors & Barrón-Meza et al. & Zong et al. \\
Reference & 8 & 9 \\
$V(\mathrm{~L})$ & 13.7 & 30.0 \\
$Q(\mathrm{~L} / \mathrm{s})$ & 0.2066 & 0.0666
\end{tabular}

Type of vessel one strand tundish continuous refining vessel Number of figure $\quad 5$ to $7 \quad 8$ to 10
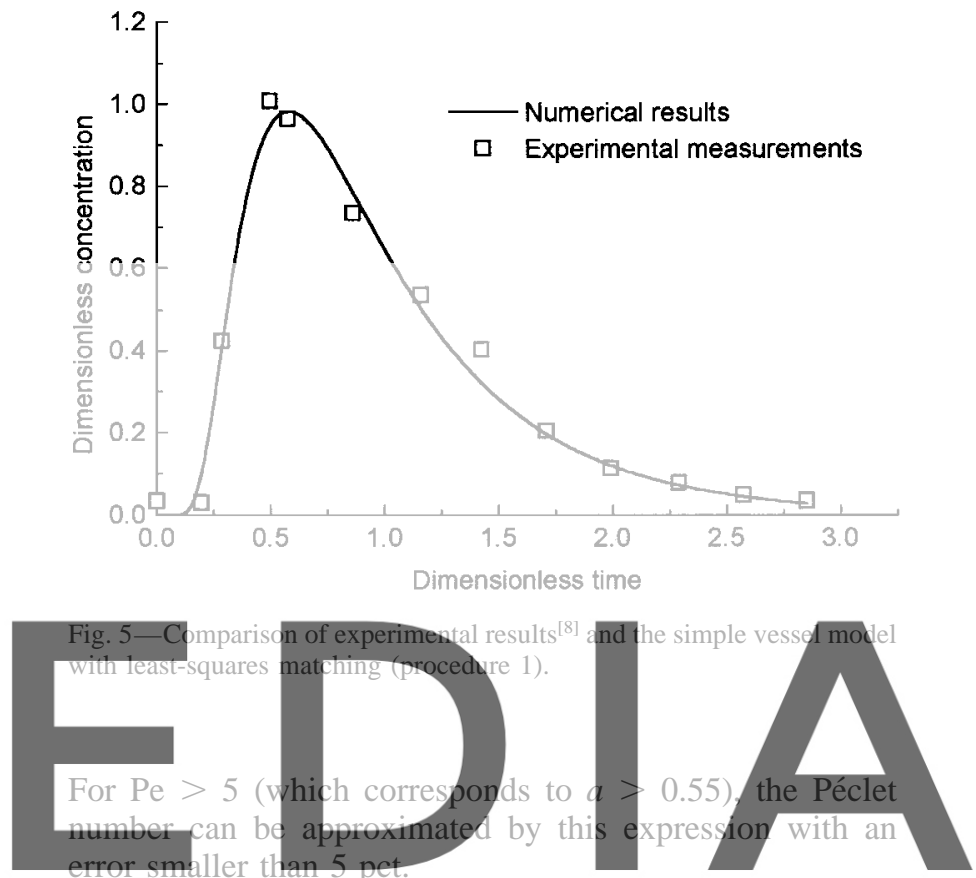

\section{dowialoadofthesversionerwithout the watermark}

The validation of the simple vessel model proposed in this section was carried out by matching two experimental RTD curves found in the literature and described in Table I.

The matching of experimental data was carried out using three different procedures to estimate the parameters Pe and $v_{a}$.

Procedure 1: Minimization of the square of the distance between the numerical results of Eq. [7] and the experimental data of the RTD curves, using the Levenberg-Marquardt algorithm. Figures 5 and 8 show the results for this procedure for the two cases described in Table I.

Procedure 2: Numerical estimation of the average residence time using the experimental data and evaluation of Pe and $v_{a}$ using Eqs. [10] and [9]. The resulting RTD curves are presented in Figures 6 and 9 for both cases.

Procedure 3: Evaluation of the product $\theta_{p} C_{p}$ from the RTD curve and calculation of Pe from Figure 4 or from Eq. [11] (in this case, a nonlinear equation must be solved using, for example, a Newton-Raphson technique) or using the asymptotic expression Eq. [12]. Finally, $v_{a}$ is obtained from Eq. [8]. Results are shown in Figures 7 and 10.

The values Pe and $v_{a}$ obtained with the different procedures for both cases are shown in Table II. The procedure 1 provides the best fitting but involves the solution of a nonlinear minimization problem. 


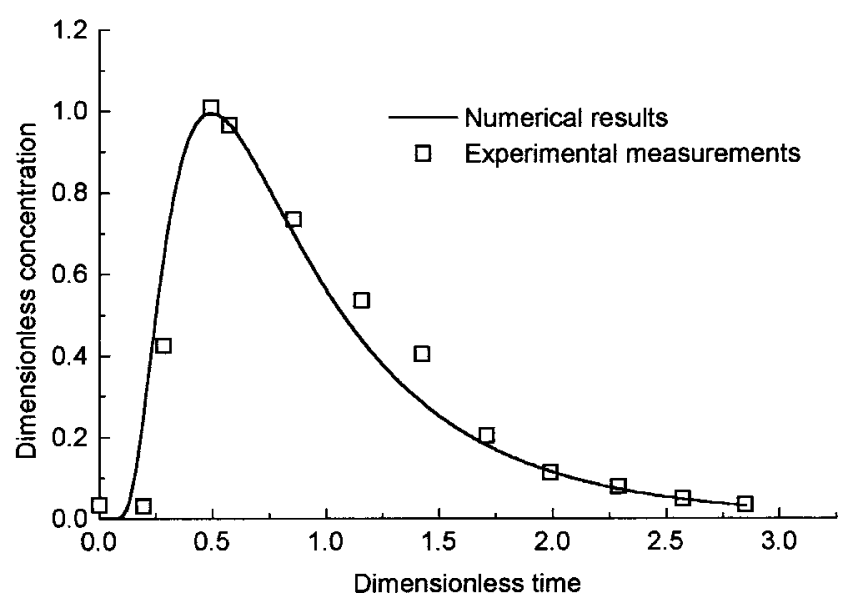

Fig. 6-Comparison of experimental results ${ }^{[8]}$ and the simple vessel model using Eqs. [10] and [9] (procedure 2).

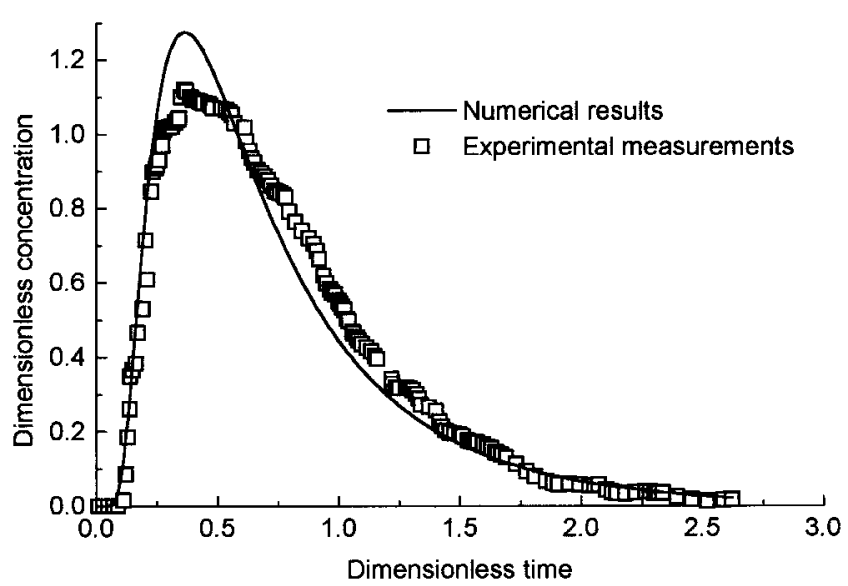

Fig. 9-Comparison of experimental results ${ }^{[9]}$ and the simple vessel model using Eqs. [10] and [9] (procedure 2).

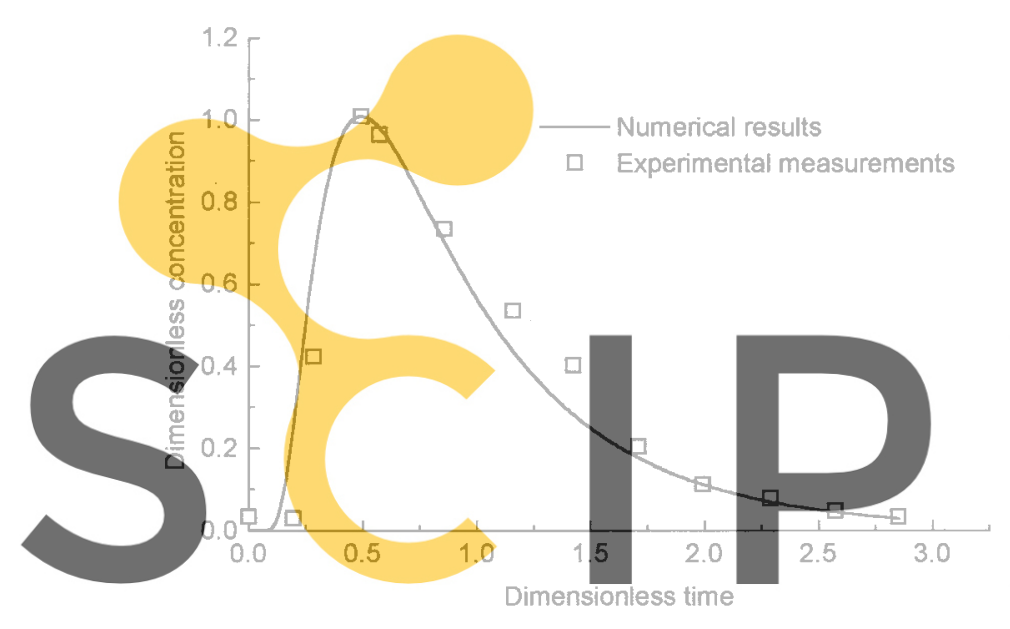

Registerg. 7-Comparison of experimental results ${ }^{[8]}$ and the simple vessel model

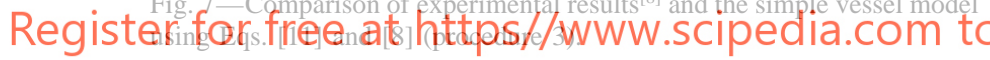

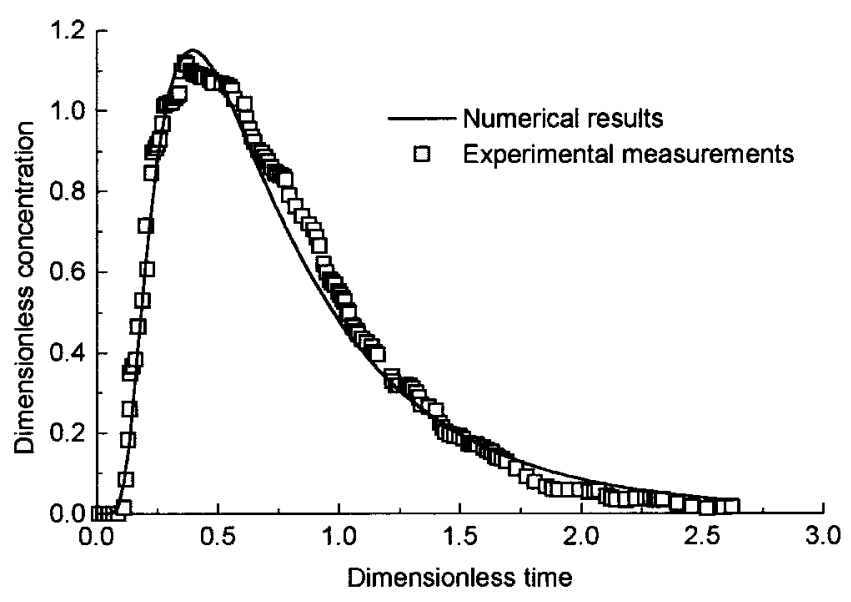

Fig. 8-Comparison of experimental results ${ }^{[9]}$ and the simple vessel model with least-squares matching (procedure 1).

Procedures 2 and 3 give a reasonable estimation of the parameters with very little information from the experimental data. The accuracy of these two procedures depend

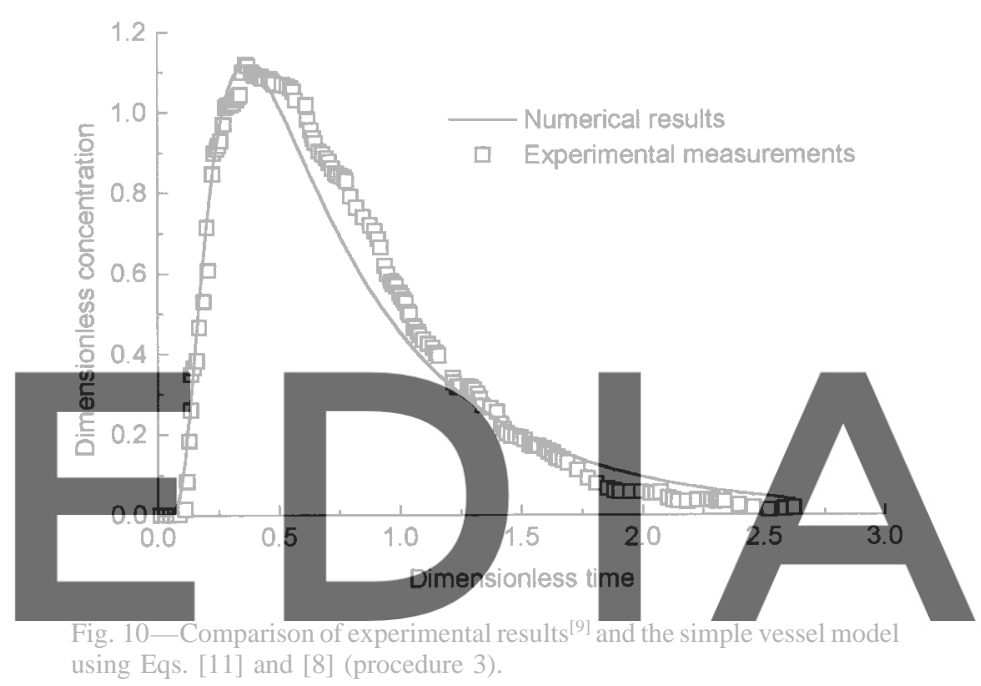

download the version without the watermark

Table II. Numerical Values of the Parameters Pe and $v_{a}$

\begin{tabular}{cccc}
\hline Procedure & Parameter & Case 1 & Case 2 \\
\hline 1 & $\mathrm{Pe}$ & 5.26 & 3.64 \\
1 & $v_{a}$ & 1.0 & 0.84 \\
2 & $\mathrm{Pe}$ & 4.11 & 3.77 \\
2 & $v_{a}$ & 0.97 & 0.76 \\
3 & $\mathrm{Pe}$ & 4.198 & 3.02 \\
3 & $v_{a}$ & 0.96 & 0.88 \\
\hline
\end{tabular}

strongly on the reliability of the evaluation of $\theta_{p}$ and $\theta_{a v}$ or $C_{p}$, from the experimental RTD curve.

\section{A MULTIVOLUME VESSEL MODEL}

The model described previously succeeded in representing a variety of experimental RTD curves. However, it fails to describe correctly RTD curves with two peaks. These curves arise when short circuits are present in the system. ${ }^{[10]}$ Consequently, a model consisting of two convection-diffusion volumes connected in parallel and a dead volume is proposed (Figure 11).

For a steady-state problem, where both the flow rate at the entrance of the vessel, $Q_{i n}$, and the flow rate at the 


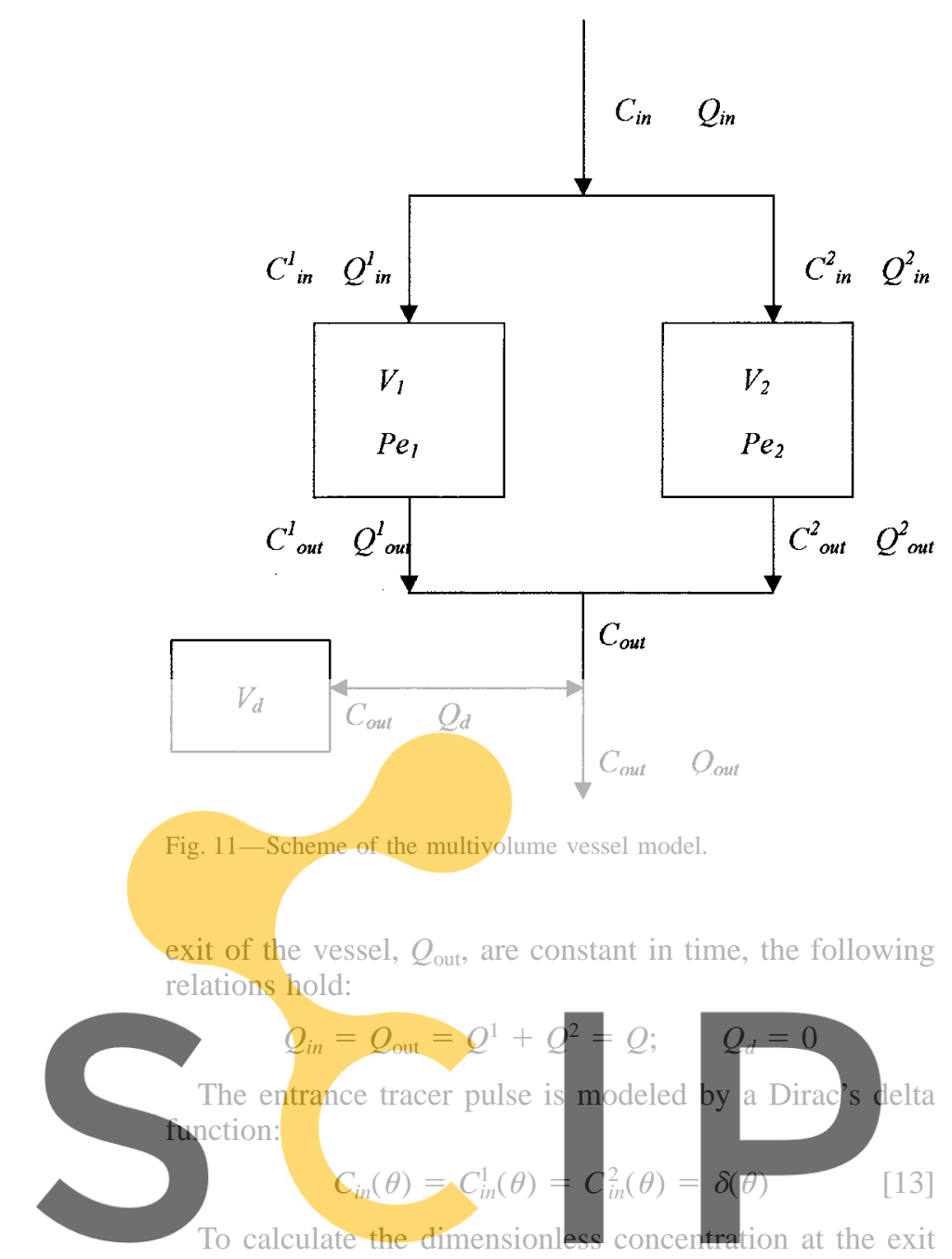

of each of the convection-diffusion volumes, Eq. [3] must

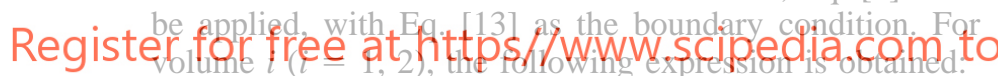

$$
C_{\text {out }}^{i}(\theta)=\frac{q^{i}}{\mathbf{f}_{V}^{i}} K_{\mathrm{Pe}^{i}}\left(1, \theta \frac{q^{i}}{f_{V}^{i}}\right)
$$

where the relative flow rate $q^{i}=Q^{i} / Q$ and the volume fraction $f_{V}^{i}=V^{i} / V_{T}$ were introduced.

Finally, the concentration exiting the system is obtained by tracer conservation, which leads to the following expression:

$$
\begin{aligned}
C_{\text {out }}(\theta) & =\frac{\left(q^{1}\right)^{2}}{f_{V}^{1}} K_{\operatorname{Pe}^{1}}\left(1, \theta \frac{q^{1}}{f_{V}^{1}}\right) \\
& +\frac{\left(q^{2}\right)^{2}}{f_{V}^{2}} K_{\mathrm{Pe}^{2}}\left(1, \theta \frac{q^{2}}{f_{V}^{2}}\right)
\end{aligned}
$$

This expression contains five dimensionless parameters, $f_{V}^{1}, f_{V}^{2}, q^{1}, \mathrm{Pe}^{1}$, and $\mathrm{Pe}^{2}$ (the parameter $q^{2}$ is given by $q^{2}=$ $\left.1-q^{1}\right)$. All the parameters must be positive and the volume fractions must satisfy the inequality $f_{V}^{1}+f_{V}^{2} \leq 1$. Note that the dead volume influences the result indirectly by reducing the convection-diffusion region (otherwise, $f_{V}^{1}+f_{V}^{2}=1$ ).

Any experimental RTD curve is expected to be well represented by Eq. [14] if suitable values for the parameters are chosen. A numerical code (RESIDENCE ${ }^{[11]}$ was developed to find the set of parameters that minimizes (in a $L^{2}$ sense) the distance between a given experimental curve and $C_{\text {out }}(\theta)$.
This program was codified in Fortran and makes use of the IMSL subroutine DBCLSF, which solves nonlinear leastsquares problems using a modified Levenberg-Marquardt algorithm. ${ }^{[12]}$

\section{Validation of a Multivolume Vessel Model}

In order to validate the multivolume model, several experimental measurements found in the literature are going to be considered. In Table III, we present the different cases to be analyzed.

In Table IV, we show the optimal values obtained by our program RESIDENCE, ${ }^{[1]}$ and in Figures 12 to 16 , we compare numerical results and experimental measurements.

Case 1 (Figure 12) has already been considered in the previous section and was matched with the simple model. From Table IV, we see that the addition of a convection diffusion volume does not affect the results. Only one convection-diffusion volume was really needed, since both $f_{V}^{1}$ and $q^{1}$ vanish.

Case 2 (Figure 13) has also been addressed in Section III. However, in this case, an improvement in the accuracy of the approximation was achieved by the multivolume model.

The third example, taken from Chakraborty and Sahai, ${ }^{[13]}$ is plotted in Figure 14. For this RTD curve, a traditional analysis becomes troublesome since numerical integration of the experimental data renders $\theta_{a 1}>1$. However, the

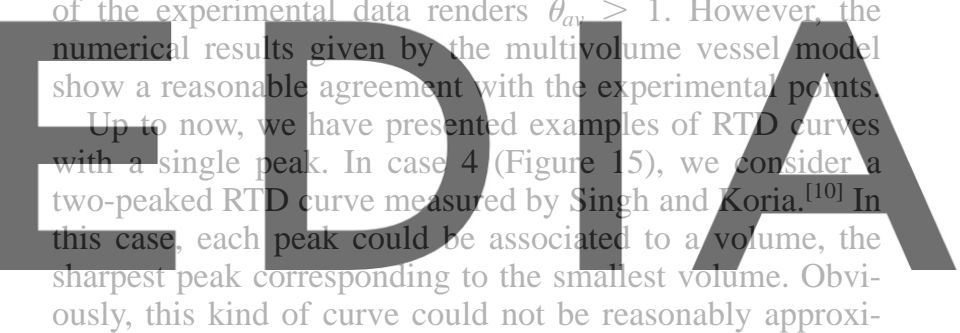

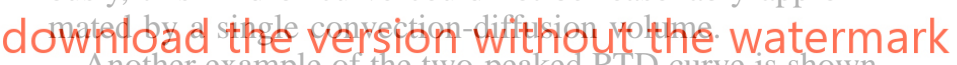
Another example of the two-peaked RTD curve is shown in Figure 16. Experimental points were also taken from the work by Zong et al. ${ }^{[9]}$ (with the water model described in the second example). Like the previous example, the extremely sharp peak due to a short-circuit is modeled by a very small convection-diffusion volume, $V_{1}$, with a high Péclet number.

\section{ANALYSIS OF A MULTIPLE LINE TUNDISH}

We present an example corresponding to a four-line tundish with the volume of $3.44 \mathrm{~m}^{3}$ and a flow rate of 49.94 $\mathrm{L} / \mathrm{min}$ in each line. In a multiple line tundish, it is of interest to model the RTD curve resulting from the addition of the RTD curves of each line. ${ }^{[1]}$

In this example, the RTD curves were obtained by the following procedure:

(1) The liquid steel flow inside the tundish was calculated with a three-dimensional numerical model using the $(k-L)$ predictor/ $(\varepsilon)$-corrector turbulent model (where $k$ is the turbulent kinetic energy, $\varepsilon$ is the dissipation rate of $k$, and $L$ is the mixing length). This numerical model was developed and tested in our previous publication. ${ }^{[14-19]}$

(2) Once the velocity field and turbulence variables were 
Table III. Different RTD Curves Considered for the Validation of the Model

\begin{tabular}{clrll}
\hline Case & \multicolumn{1}{c}{ Authors } & $V(\mathrm{~L})$ & $Q(\mathrm{~L} / \mathrm{s})$ & \multicolumn{1}{c}{ Vessel Type } \\
\hline 1 & Barrón Meza et al.$^{[8]}$ & 13.7 & 0.2066 & one strand tundish \\
2 & Zong et al..$^{[9]}$ & 30.0 & 0.0666 & continuous refining vessel \\
3 & Chakraborty and Sahai ${ }^{[13]}$ & 186.3 & 0.5046 & one strand tundish \\
4 & Singh and Koria ${ }^{[10]}$ & 86.2 & 0.155 & one strand tundish \\
5 & Zong et al. ${ }^{[9]}$ & 30.0 & 0.0666 & continuous refining vessel \\
\hline
\end{tabular}

Table IV. Optimal Values of the Parameters

\begin{tabular}{clllrl}
\hline Cases & \multicolumn{1}{c}{$f_{v}^{1}$} & $f_{v}^{2}$ & \multicolumn{1}{c}{$q^{1}$} & $\mathrm{Pe}_{1}$ & $\mathrm{Pe}_{2}$ \\
\hline 1 & 0.00 & 1.00 & 0.00 & 1.00 & 5.26 \\
2 & 0.23 & 0.54 & 0.23 & 12.12 & 4.00 \\
3 & 0.29 & 0.71 & 0.42 & 3.03 & 5.63 \\
4 & 0.87 & 0.025 & 0.87 & 3.59 & 6.64 \\
5 & 0.0083 & 0.76 & 0.045 & 223.7 & 4.34 \\
\hline
\end{tabular}

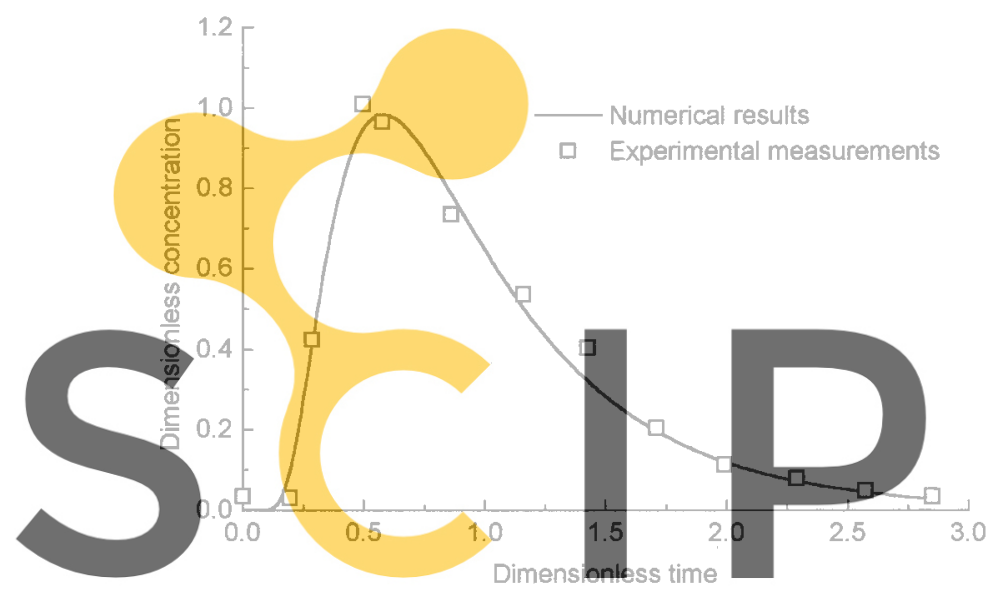

Fig. 12-Comparison of experimental results ${ }^{[8]}$ and the multivolume vesRegister folpl.free at https//www.scipedia.com to

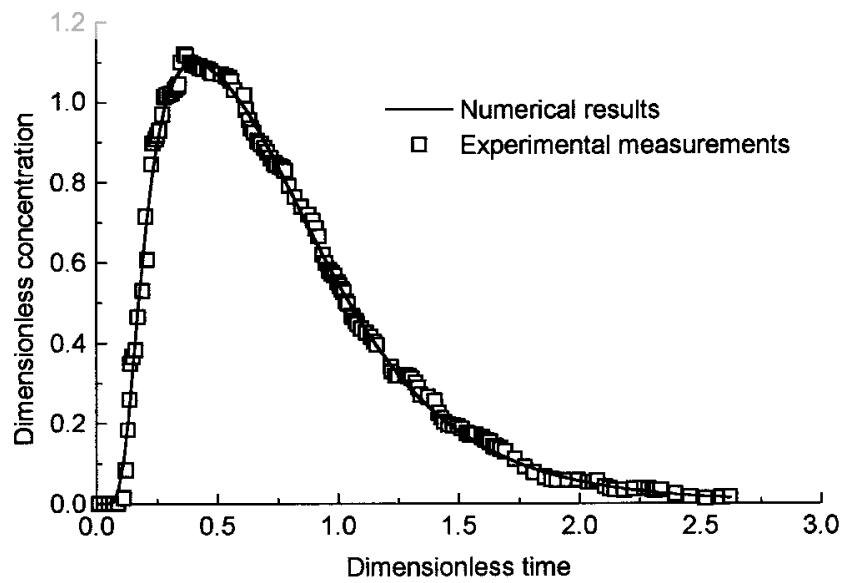

Fig. 13 -Comparison of experimental results ${ }^{[9]}$ and the multivolume vessel model.

obtained, the tracer transport equation in a turbulent stream was calculated by solving a transient threedimensional turbulent convection-diffusion equation. ${ }^{[20]}$

In Figure 17, the internal and external lines of a symmetric

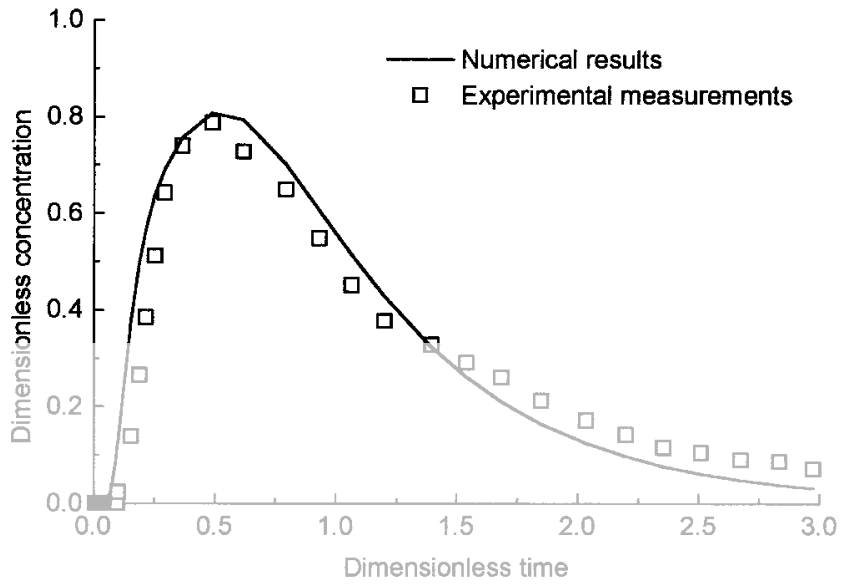

Fig. 14-Comparison of experimental results ${ }^{[13]}$ and the multivolume vessel model.

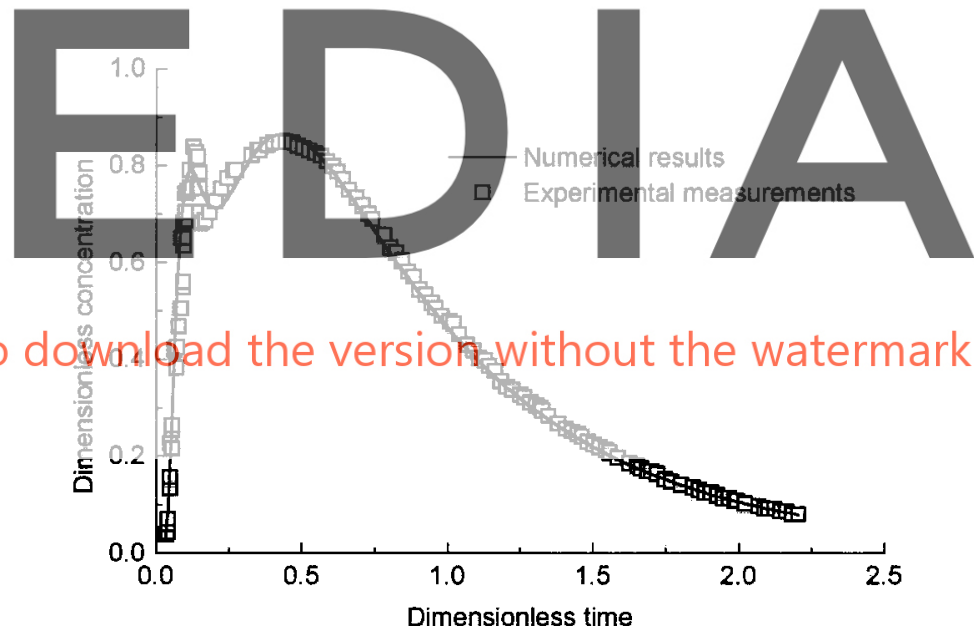

Fig. 15-Comparison of experimental results ${ }^{[10]}$ and the multivolume vessel model.

four-line tundish are shown, together with the global RTD curve. The approximation of the global RTD curve, also shown in Figure 17, is obtained using the multiple volume model described in Section IV.

The values of the parameter obtained for this case are $f_{V}^{1}=0.56, f_{V}^{2}=0.36, q^{1}=0.54, \mathrm{Pe}_{1}=9.01$, and $\mathrm{Pe}_{2}=1.96$.

\section{CONCLUSIONS}

Two numerical models for the simulation of RTD curves in different vessels are presented. The comparison of measured RTD curves with numerical results from the proposed models shows that these models can successfully represent the general behavior of the fluid inside a variety of systems. 


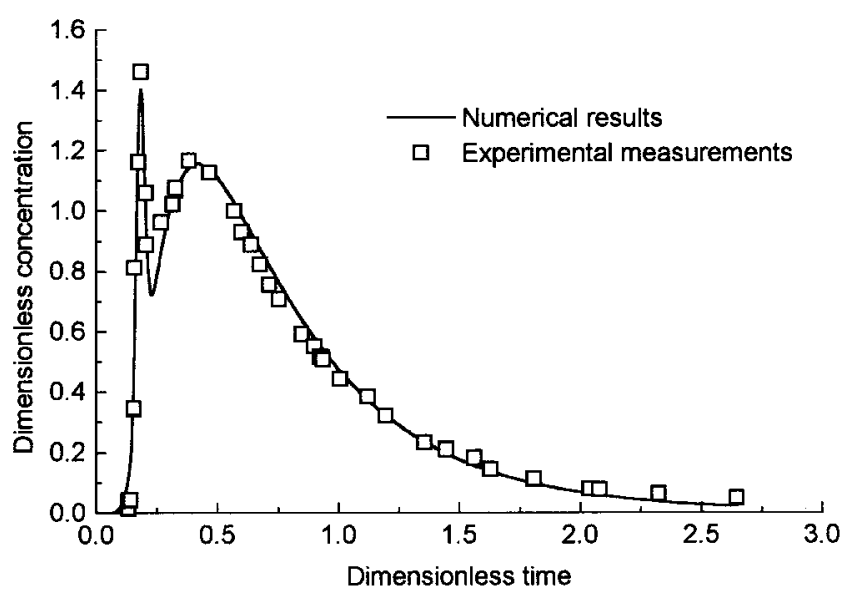

Fig. 16-Comparison of experimental results ${ }^{[9]}$ and the multivolume vessel model.

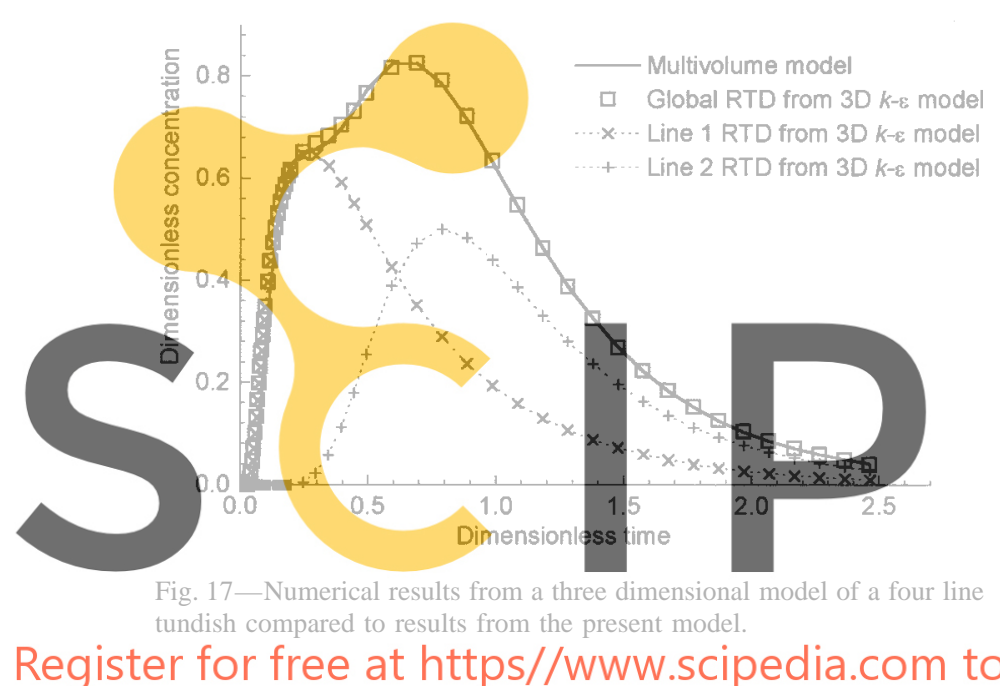

The RTD curves used for validation of the model include experimental data found in literature and numerical data obtained from a full three-dimensional computation of the turbulent flow in a tundish.

The first of the models proved to be efficient to describe most of the one-peaked RTD curves, in spite of its simplicity. The second one, slightly more complex, represented successfully all the different RTD curves under consideration, including those with two peaks.

The key feature of these models is the use of a new type of volume, the convection-diffusion volume introduced in this work. The characteristics of this volume were deduced from the convection-diffusion one-dimensional equation with a pulse boundary condition in the origin. For this reason, the use of convection-diffusion volume is not restricted to systems that exhibit a small degree of mixing. This allows the representation of the different vessels with very simple models.

In order to find the parameters of the model for a given experimental RTD curve, a numerical algorithm was developed. We also found some simple mathematical relations that allow the estimation of the parameters of the model from the characteristic parameters of the RTD curve.

\section{LIST OF SYMBOLS}

$a(\mathrm{Pe})$

C

$C_{0}$

$C_{p}$

$D$

$f_{V}^{i}$

$K_{\mathrm{Pe}}$

$\hat{K}_{\mathrm{Pe}}$

$L$

$\mathrm{Pe}$

$q^{i}$

$Q$

V

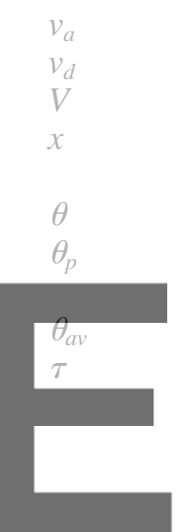

The authors thank Dr. E. Dvorkin for his continuous sup port in numerical methods and Dṛ. Javier Etcheverry for his

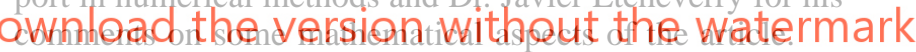

This research was supported by SIDERCA (Campana, Argentina), SIDOR (Puerto Ordaz, Venezuela), and DALMINE (Bérgamo, Italy).

\section{REFERENCES}

1. D. Mazumdar and R.I.L. Guthrie: Iron Steel Inst. Jpn. Int., 1999, vol. 39, pp. 524-47.

2. B.E. Launder and D.B. Spalding: Comp. Meth. Appl. Mech. Eng. 1974, vol. 3, pp. 269-89.

3. J. Szekely and N.J. Themelis: Rate Phenomena in Process Metallurgy, John Wiley \& Sons Inc., New York, NY, 1971, pp. 515-55.

4. A.D. Martin: Chem. Eng. Sci., 2000, vol. 6, pp. 5907-17.

5. Y. Sahai and R. Ahuja: Ironmaking and Steelmaking, 1986, vol. 13, pp. 241-52.

6. O. Levenspiel and W.K. Smith: Chem. Eng. Sci., 1957, vol. 6, pp. 227-33.

7. O. Levenspiel: Chemical Reaction Engineering, John Wiley \& Sons Inc., New York, NY, 1972, pp. 277-347.

8. M.A. Barrón-Meza, J. de J. Barreto-Sandoval, and R.D. Morales: Metall. Mater. Trans. B, 2000, vol. 31B, pp. 63-74.

9. J. Zong, K. Yi, and J. Yoon: Iron Steel Inst. Jpn. Int., 1999, vol. 39, pp. 139-48.

10. S. Singh and S. Koria: Steel Res., 1995, vol. 66, p. 294.

11. RESIDENCE User Manual, Center for Industrial Research, Buenos Aires, 2001.

12. IMSL: IMSL MATH/LIBRARY User's Manual, Version 3.0, Visua Numerics, Inc., Houston, TX, 1994. 
13. S. Chakraborty and Y. Sahai: Metall. Trans. B, 1991, vol. 22B, pp 429-37.

14. M.B. Goldschmit and M.A. Cavaliere: Appl. Mech. Rev., ASME, 1995, vol. 48 (11), part 2, pp. S211-S215.

15. M.B. Goldschmit and M.A. Cavaliere: Eng. Computations, 1997, vol. 14 (4), pp. 441-55.

16. M.B. Goldschmit, R.J. Principe, and M. Koslowski: 3rd Eur. Conf on Continuous Casting, Madrid, Oct. 1998.
17. M.B. Goldschmit: 80th Steelmaking Conf., EEUU, Chicago, IL, 1997.

18. M.B. Goldschmit, R.J. Principe, and M. Koslowski: Int. J. Num. Meth. Eng., 1999, vol. 46, pp. 1505-19.

19. M. Maldovan, J. Príncipe, G. Sánchez, A. Pignotti, and M. Goldschmit: ECCOMAS 2000, Barcelona, 2000.

20. M.B. Goldschmit, S.P. Ferro, G.F. Walter, V.G. Aranda, and J.A. Tena Morelos: Metall. Mater. Trans. B, 2001, vol. 32B, pp. 537-46.
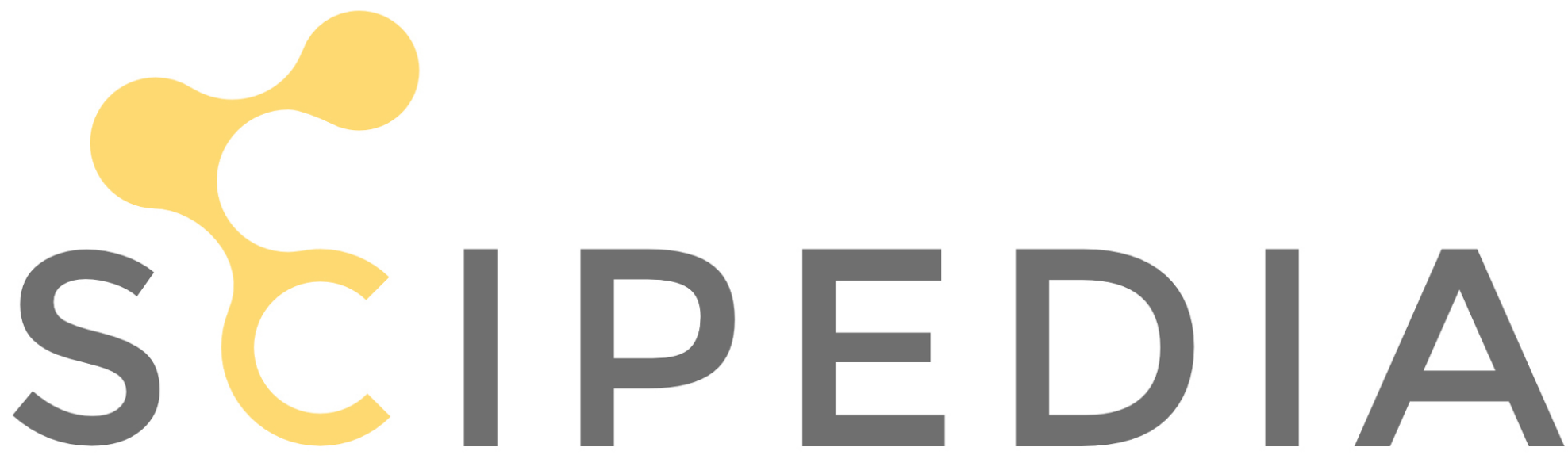

Register for free at https//www.scipedia.com to download the version without the watermark 